PREPARED FOR THE U.S. DEPARTMENT OF ENERGY, UNDER CONTRACT DE-AC02-76CH03073

PPPL-3722

PPPL-3722

UC-70

Correlation Reflectometry for Turbulence and Magnetic Field Measurements in Fusion Plasmas

by

G.J. Kramer, R.Nazikian, and E. Valeo

July 2002

$\left.\stackrel{M}{M}\right|_{\substack{\text { PRInCETON PLASIMA } \\ \text { PHYSICS LABORATORY }}} ^{D}$

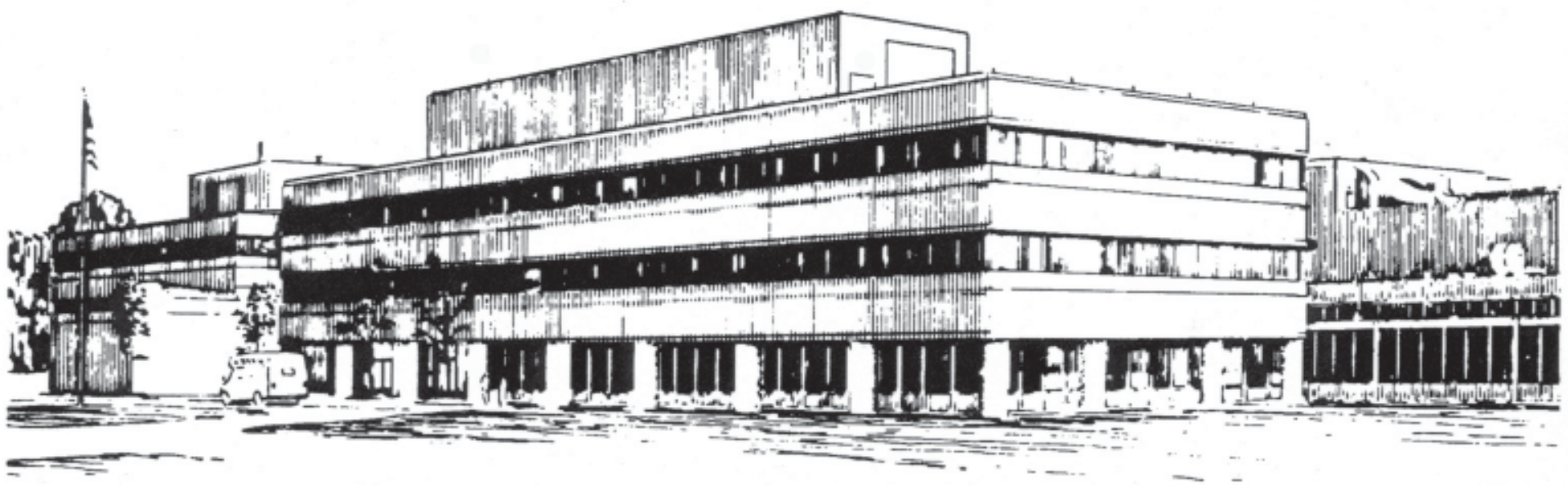

PRINCETON PLASMA PHYSICS LABORATORY PRINCETON UNIVERSITY, PRINCETON, NEW JERSEY 


\section{PPPL Reports Disclaimer}

This report was prepared as an account of work sponsored by an agency of the United States Government. Neither the United States Government nor any agency thereof, nor any of their employees, makes any warranty, express or implied, or assumes any legal liability or responsibility for the accuracy, completeness, or usefulness of any information, apparatus, product, or process disclosed, or represents that its use would not infringe privately owned rights. Reference herein to any specific commercial product, process, or service by trade name, trademark, manufacturer, or otherwise, does not necessarily constitute or imply its endorsement, recommendation, or favoring by the United States Government or any agency thereof. The views and opinions of authors expressed herein do not necessarily state or reflect those of the United States Government or any agency thereof.

\section{Availability}

This report is posted on the U.S. Department of Energy's Princeton Plasma Physics Laboratory Publications and Reports web site in Fiscal Year 2002. The home page for PPPL Reports and Publications is: http://www.pppl.gov/pub_report/

DOE and DOE Contractors can obtain copies of this report from:

U.S. Department of Energy

Office of Scientific and Technical Information

DOE Technical Information Services (DTIS)

P.O. Box 62

Oak Ridge, TN 37831

Telephone: (865) 576-8401

Fax: (865) 576-5728

Email: reports@adonis.osti.gov

This report is available to the general public from:

National Technical Information Service

U.S. Department of Commerce

5285 Port Royal Road

Springfield, VA 22161

Telephone: 1-800-553-6847 or

(703) 605-6000

Fax: (703) 321-8547

Internet: http://www.ntis.gov/ordering.htm 


\title{
Correlation Reflectometry for Turbulence and Magnetic Field Measurements in Fusion Plasmas
}

\author{
G.J. Kramer, R. Nazikian, E. Valeo \\ Princeton Plasma Physics Laboratory, Princeton NJ 08543-0451, USA
}

\begin{abstract}
For the interpretation of correlation reflectometry data a fast two dimensional full wave code has been developed in which realistic plasma geometries are used. Results of this code are compared with experiments and turbulence correlation lengths and fluctuation levels are extracted with statistical optics methods. It is shown that in general the measured reflectometer correlation length is not equal to the turbulence correlation length. The code is also used to study the posability of O-X correlation reflectometry in FIRE for the determination of the local magnetic field strength. It was found that this is only possible at very low fluctuation levels.
\end{abstract}

\section{1 introduction}

Correlation reflectometry has been performed for many years in both laboratory and fusion scale plasmas $[1,2,3,4,5]$. The aim of the laboratory scale experiments with its easy access for probes, is to compare fluctuation levels and correlation lengths deduced from reflectometer measurements with those from probe measurements. In fusion scale experiments, however, probe measurements in the core of the plasma for comparison with reflectometer measurements are no longer feasible, so the results from laboratory scale experiments have to be extrapolated to fusion scale experiments.

For this purpose we have developed a very fast 2-D reflectometer simulation code with realistic plasma geometry that can be used to study correlation reflectometry in detail [6]. With this model we can answer how robust the 1-D and slab geometry estimates $[7,8]$ of the fluctuation levels and correlation lengths are to details of the plasma 
profile and geometry. Slab models and 1-D models do not treat amplitude fluctuations, plasma curvature, finite aperture and alignment effects correctly.

We will present results of 2-D laboratory and fusion scale plasma simulations, compare it with experiments and 1-D models and show that 2-D effects are important in some cases for the interpretation of reflectometer experiments.

Correlations between $\mathrm{O}$ - and X-mode polarized signals can be used to measure local magnetic field strengths in plasmas [9]. We will also present results of a 2-D O-X correlation simulation and show that there is a good agreement with laboratory scale experiments. Extrapolation of these results to fusion devices show that it is far from certain that this technique will give reliable measurements of the local magnetic field in high field devices because of the very low fluctuation levels that are needed to obtain a significant correlation between the $\mathrm{O}$ - and $\mathrm{X}$-mode signals.

\section{Interpretation of correlation measure- ments}

From correlation reflectometry two important quantities for the study of plasma turbulence and transport can be deduced. These are the turbulence correlation length, $\lambda_{T}$, and the density fluctuation level, $\tilde{n} / n$.

For correlation reflectometry two microwave beams with different frequencies are launched into the plasma and the cross correlation between the two reflected signals is studied (see figure 1). The two waves can either be launched in X-mode, usually to study the plasma core, O-mode, for edge measurements, or one channel in X-mode and the other in $\mathrm{O}$-mode for the determination of the local magnetic field strength. In section 4, O-X correlations are studied in detail to obtain local magnetic field measurements in plasmas.

O-mode waves are reflected at the plasma frequency cut off layer which depends on the local electron density whereas X-mode waves are reflected at the left- and right-hand cut off layers which depend on the local electron density and magnetic field strength. The radial separation between the two channels is obtained from the frequency difference and the density, and in the X-mode case, magnetic field profiles. These profiles can be determined from other diagnostics. 
Instead of studying the amplitude and phase fluctuations of the reflected signals separately, we have chosen to use statistical optics methods [10] for both the experimental and simulated data. It has been shown that the amplitude fluctuations in the reflected signal are introduced by interference effects of the scattered waves [11].

From the correlation reflectometer signals we can calculate two signals that are important for the interpretation the measurements: the normalized cross correlation, $\gamma$, and the coherent reflection, $G$. The normalized cross correlation is defined as

$$
\gamma=\frac{\left|\left\langle E_{1} E_{2}^{*}\right\rangle\right|}{\sqrt{\left\langle\left|E_{1}\right|^{2}\right\rangle\left\langle\left|E_{2}\right|^{2}\right\rangle}}
$$

with $E_{1}$ and $E_{2}$ the (complex) signal of reflectometer channel 1 and 2 , respectively, and $\langle\cdots\rangle$ stands for ensemble averaging. The normalized cross correlation can be used to estimate the radial correlation length of the turbulence. Until now the 1/e width of the measured cross correlation distribution is used as a measurement of the radial turbulence correlation length but in the following we will show that in general the radial correlation length as measured with a reflectometer is not equal to the turbulence correlation length.

The coherent reflection is given by

$$
G=\frac{|\langle E\rangle|}{\sqrt{\left\langle|E|^{2}\right\rangle}}
$$

and can be used as an indicator of the density fluctuation level.

In the following we study the relationship between the experimentally determined $\gamma$ and $G$ and the theoretical quantities $\lambda_{T}$ and $\tilde{n} / n$ and we show that both $\gamma$ and $G$ are needed together with 2-D (or 1-D) modeling to determine $\lambda_{T}$ and $\tilde{n} / n$ uniquely.

\section{Turbulence simulations}

Correlation reflectometry has been performed in the edge of laboratory scale plasmas [3] and in the core of large scale fusion devices $[2,12]$. In some of those experiments the measured reflectometer correlation length is interpreted as the turbulence correlation length. In this section we present exhaustive 1-D and 2-D reflectometer modeling and show that in general these two correlation lengths are different. We will also show a benchmark of the 1-D and 2-D codes with an experiment performed at the LArge Plasma Device [13]. 
We have simulated the behavior of microwave reflection from the edge of a plasma with a 1-D [8] and a 2-D code [6]. In our calculations we have taken a cylindrical plasma, radius $60 \mathrm{~cm}$, a density scale length of $10 \mathrm{~cm}$, and $\mathrm{O}$-mode polarized waves with a frequency of $12 \mathrm{GHz}$. These waves were reflected from a layer $10 \mathrm{~cm}$ inside the plasma. On top of the equilibrium density profile a spectrum of density fluctuations was added which gives the following density correlation function

$$
\left\langle\tilde{n}\left(x_{2}\right) \tilde{n}\left(x_{1}\right)\right\rangle / n^{2}=\left(\frac{\tilde{n}}{n}\right)_{x_{1}}^{2} \exp \left(-\left(x_{2}-x_{1}\right)^{2} / \lambda_{T}^{2}\right) \cos \left(k_{\mathrm{fl}}\left(x_{2}-x_{1}\right)\right)
$$

with $(\tilde{n} / n)_{x_{1}}$ the fluctuation amplitude, $x_{1}\left(x_{2}\right)$ the fixed (variable) frequency cut off layer position, $\lambda_{T}$ the $1 / \mathrm{e}$ width of the turbulence correlation and $k_{\mathrm{fl}}=2 \pi / \lambda_{\mathrm{fl}}$ where $\lambda_{\mathrm{fl}}$ is the characteristic fluctuation wave length. In a similar manner a 2-D spectrum of density fluctuations (spectral locations $k_{x}$ and $k_{y}$ and widths $\Delta k_{x}$ and $\Delta k_{y}$ ) was added to the $2-\mathrm{D}$ density equilibrium profile for the 2 -D calculations [6].

In our simulations we have varied the theoretically important variables $\tilde{n} / n$ between 0.1 and $12 \%$ in steps of $1 \%$ and $\lambda_{T}$ between 0.5 and 2.5 in steps of 0.5 times the width of the Airy fringe at the cut off layer which is given (for O-mode) by $w_{\text {Airy }}=0.48 L_{n}^{1 / 3} \lambda_{0}^{2 / 3}$.

The 1-D correlations were calculated on 401 equally spaced radial points over an interval of six correlation lengths. The radial grid for the 2-D simulations was an equally spaced grid of 13 points with a spacing of $0.5 \mathrm{~cm}$. For the 1-D calculations a set of 6000 random density distributions, $\tilde{n}(x)$, were generated from the correlation function whereas for the $2-\mathrm{D}$ simulations an ensemble of 300 realizations was used.

In figure 2 the relation is shown between $\lambda_{T}$ and the inferred reflectometer correlation length, $\lambda_{R}$, at different density fluctuation levels. It can be seen that in general $\lambda_{T}$ is not equal to $\lambda_{R}$. At low fluctuation levels and short turbulence correlation lengths, $\lambda_{R}$ is larger than $\lambda_{T}$ whereas at high fluctuation levels and long turbulence correlation lengths $\lambda_{R}$ is smaller than $\lambda_{T}$.

When one measures only $\lambda_{R}$, the corresponding $\lambda_{T}$ is not determined uniquely, because of the $\tilde{n} / n$ dependence. We can resolve that ambiguity by not only measuring $\lambda_{R}$ but also the coherent reflected power, $G$, which is correlated with $\tilde{n} / n$. A clear way to represent this, is to map the quantities of the turbulence, $\left(\lambda_{T}, \tilde{n} / n\right)$, to the $\left(\lambda_{R}, G\right)$ 
plane, which are both experimental quantities, as shown in figure 3. First of all, there is a remarkable similarity between the 1-D and 2D simulation results. From figure 3 it can also be seen that if $\tilde{n} / n$ increases at constant $\lambda_{T}, G$ decreases. $G$ also decreases when $\tilde{n} / n$ is kept constant and $\lambda_{T}$ is increased. In a similar way $\lambda_{R}$ decreases when $\tilde{n} / n$ increases at constant $\lambda_{T}$.

We conclude from figure 3 that it is required to measure both $\lambda_{R}$ and $G$ to determine $\lambda_{T}$ and $\tilde{n} / n$. Determining $\lambda_{R}$ alone as a measure of $\lambda_{T}$, leaves one with a range of possible $\lambda_{T}$ 's.

The relation between correlation lengths determined from probes as a measure of the turbulence correlation length and the correlation lengths from reflectometer measurements has been studied exhaustively in a laboratory scale plasma at LAPD [3]. We have used the data of one of those measurements in our 1-D code as a benchmark. We have used the 1-D instead of the 2-D code because the the two codes give the same results for the parameter range that is covered in this simulation as was shown in figure 3 . In our benchmark calculation we have used $1.7 \mathrm{~cm}$ for the turbulence correlation as was measured with the probes. Other experimental parameters that were used are a density scale length of $12 \mathrm{~cm}, 14 \mathrm{GHz}$ X-mode polarized waves, and a magnetic field of $0.1 \mathrm{~T}$. In our simulations we have varied the density fluctuation level between 5 and $19 \%$ and found that the $9 \%$ fluctuation level which was measured with the probes, fits the measured 1 /e reflectometer width very well (see figure 4 ). In a separate study where measured $\mathrm{O}-\mathrm{X}$ cross correlations were compared with 1-D and 2-D modeling and where 2-D effects were shown to be important, good agreement with the modeling and experiments was also found. Details of those simulations were reported in [8].

We conclude that simulations agree well with experimental results. A study is under way in which the the 1-D and 2-D codes are used to analize all the experimental and model sensitivities for reflectometer data that was measured in the core of a large scale fusion plasma, inside the internal transport barrier of a JT-60U plasma $[2,12]$

\section{Magnetic field measurements}

Magnetic field measurements are essential for fusion devices. In the next generation of Tokamaks it will become very difficult to use Motional Stark Effect (MSE) measurements for the determination of the 
magnetic safety factor or $q$-profile $[14,15]$ because of the expected high plasma densities in which diagnostic neutral beams cannot penetrate to the plasma center.

In two recent articles $[3,4]$ an alternative approach to magnetic field measurements in hot plasmas has been proposed based on the reflection of microwaves of different polarizations from magnetized plasmas. In this method the omnipresent turbulence in the plasma is used by measuring the peak correlation in the reflected signal between ordinary or O-mode and extraordinary or X-mode polarized waves. The absolute value of the magnetic field, $|\mathbf{B}|$, at the reflection point can then be deduced from the electron cyclotron frequency, $\omega_{c}$, and the measured O-mode, and right hand side $\mathrm{X}$-mode frequencies, $\omega_{O}$ and $\omega_{R}$,

$$
\omega_{c}=\frac{-e|\mathbf{B}|}{m_{e}}=\frac{\omega_{R}^{2}+\omega_{O}^{2}}{\omega_{R}}
$$

with $e$ and $m_{e}$ represent the electron charge and mass respectively. In LAPD O-X correlations have been studied extensively [3, 4] with uniform magnetic fields between 0.1 and $0.18 \mathrm{~T}$, densities up to $3.010^{18} \mathrm{~m}^{-3}$, and density scale lengths between 0.05 and $0.20 \mathrm{~m}$. In these experiments, in which the X-mode frequency was scanned and the O-mode frequency kept fixed, it was found that the O-X correlation was reduced with increasing magnetic field strengths, and it peaked slightly in front of where the two cut off layers coincide. Both effects have been explained successfully with 2-D modeling [8].

Future burning plasma experiments such as FIRE and ITER, will operate at very high magnetic field strengths $(10 \mathrm{~T}$ and $5.3 \mathrm{~T}$, respectively), so it is not clear whether there is any $\mathrm{O}-\mathrm{X}$ correlation left at these field strengths. To answer this question, we have performed extensive 1-D and 2-D simulations for an envisaged FIRE discharge with a toroidal magnetic field of $10 \mathrm{~T}$, a central electron density of $4.910^{20} \mathrm{~m}^{-3}$, and a central electron temperature of $11.9 \mathrm{keV}$. The FIRE plasma shape has been designed to be: major radius $2.14 \mathrm{~m}$, minor radius $0.60 \mathrm{~m}$, ellipticity 2.0, and triangularity 0.49 [16].

In our 1-D and 2-D simulations we have chosen an O-mode frequency of $190.0 \mathrm{GHz}$ which is reflected at $r / a$ of 0.34 as can be seen from figure 5 . The right hand side $\mathrm{X}$-mode frequencies were scanned between 350.2 and $354.5 \mathrm{GHz}$. An isotropic spectrum of density fluctuations with a correlation length, $\lambda_{T}$, of $1.0 \mathrm{~cm}$ and fluctuation levels, $\tilde{n} / n$, of $0.1,0.3$, and $0.5 \%$ were added to the equilibrium density profile (for more details see $[6,8]$ ). The antennas, about $1.1 \mathrm{~cm}$ in height, 
were located in the simulation at the low field side mid-plane and $50 \mathrm{~cm}$ from the plasma edge.

For the 1-D calculations an ensemble size of 3000 was used. Radially in 1-D, the O-X correlation was calculated for $10 \mathrm{~cm}$ before to $10 \mathrm{~cm}$ behind the O-mode reflection layer with a radial step of $0.25 \mathrm{~mm}$. In the 2-D calculations the ensemble size was 300 and up to 25 different $\mathrm{X}$-mode frequencies were used separated radially between $1.25 \mathrm{~mm}$ and $2.50 \mathrm{~mm}$ in such a way that the peak of the cross correlation is covered accurately.

In figure 6 the results of the 1-D and 2-D simulations are shown. It is quite amazing to find a significant $\mathrm{O}-\mathrm{X}$ cross correlation in the 2-D simulations at $0.1 \%$ fluctuation level because the scattered wave fields for the two polarizations are very different as is shown in figures 7 and 8. The maximum 2-D cross correlation of 0.4 is reached $3 \mathrm{~mm}$ in front of the O-mode reflection layer which corresponds with a $\mathrm{X}$ mode frequency shift of $0.34 \mathrm{GHz}$. There is a significant difference in the magnitude of the cross correlation between the 1-D and 2-D modeling (see figure 6). This can be explained by the fact that in the 1-D modeling only the phase fluctuations are taken into account whereas in the 2-D modeling both phase and amplitude fluctuations are present in the signal at the (software) antenna.

Only at very low fluctuation levels, there is a significant correlation between the $\mathrm{O}$-mode and $\mathrm{X}$-mode signals and the $\mathrm{O}-\mathrm{X}$ correlation technique can used in the next generation of fusion devices. Moreover, the density profile should have such a shape that the O-mode waves can reach the locations where one wants to measure $|\mathbf{B}|$. Usually, this means a monotonically decreasing density profile from the center to the edge with a finite density gradient.

It should also be stressed that the fluctuation level must be low so that the coherent scattered power is significant and peak cross correlations is above the noise level as shown in figure 9. From this figure it is concluded that the fluctuation level for the FIRE equilibrium studied here should be well below $0.2 \%$ at a turbulence correlation length of $1.0 \mathrm{~cm}$. When the turbulence correlation length is smaller the cross correlation increases as was shown in section 2. Experimentally, fluctuation levels of 0.2 to $0.5 \%$ have been found in large scale fusion experiments $[12,17,18,19,20]$.

With the O-X correlation reflectometry method, the $|\mathbf{B}|$-profile is measured as a function of the electron density. for the determination of the $|\mathbf{B}|$-profile as function of minor or major radius, high spatial 
resolved and accurate measurements of the density profile are needed.

The accuracy of these magnetic field measurements can be expressed as

$$
\frac{|\Delta \mathbf{B}|}{|\mathbf{B}|}=\frac{\Delta \omega_{c}}{\omega_{c}}=\frac{\omega_{R}}{\omega_{R}^{2}+\omega_{O}^{2}}\left(\frac{2 \omega_{O}}{\omega_{R}} \Delta \omega_{O}+\frac{\omega_{R}^{2}-\omega_{O}^{2}}{\omega_{R}^{2}} \Delta \omega_{R}\right)
$$

with $\Delta \omega_{O}$ and $\Delta \omega_{R}$ the experimental uncertainties in the O-mode and $\mathrm{X}$-mode frequencies which includes the accuracy with which the peak of the cross correlation can be determined.

The change in the magnetic field in the above used FIRE equilibrium due to plasma effects is in the order of $0.25 \mathrm{~T}$ at the location where we have performed the simulation, which corresponds to a change in $\omega_{c}$ of $7 \mathrm{GHz}$. The estimated experimental uncertainties of $0.1 \mathrm{GHz}$ for the $\mathrm{O}$-mode and $0.2 \mathrm{GHz}$ for the $\mathrm{X}$-mode frequencies result in a $\Delta \omega_{c}$ of $0.25 \mathrm{GHz}$ which in turn gives $0.01 \mathrm{~T}$ accuracy for $|\Delta \mathbf{B}|$.

\section{Conclusions and further plans}

Microwave correlation reflectometry has the potential to determine turbulence correlation lengths and fluctuation levels. From 1-D and 2-D simulations and statistical optics analysis methods, it was shown that for an unambiguous determination of fluctuation levels and turbulence correlation lengths both reflectometer correlation lengths and coherent scattered powers have to be determined experimentally. It was also shown that the measured reflectometer correlation length depends both on the turbulence correlation length and on the fluctuation level. Vice versa, the coherent scattered power depends on both the density fluctuation level and the turbulence correlation length.

1-D and 2-D reflectometer simulations for experiments performed at LAPD have shown an excellent agreement with the experimental results. However, for a more stringent comparison between experiment and simulations, the coherent scattered power has to be measured in future experiments together with the reflectometer correlation length.

Under certain conditions the absolute value of the local magnetic field can be deduced from $\mathrm{O}-\mathrm{X}$ cross correlation measurements. Experimentally, this technique has been used successfully in LAPD at magnetic fields up to $0.2 \mathrm{~T}$. For a good theoretical understranding of these experiments 2-D simulations had to be performed. 1-D simulations overestimated the maximum cross correlation significantly. We 
have used the 2-D simulation code to study the feasibility to use O-X cross correlations in a FIRE like plasma for local internal magnetic field measurements near the core. It was found that only at very low fluctuation levels, less than $0.2 \%$, and short turbulence correlation lengths, $1 \mathrm{~cm}$ or less, sufficient high $\mathrm{O}-\mathrm{X}$ cross correlation exists so that this technique can be used successfully there. In the low field spherical tokamak NSTX experiments are underway to use this technique as a local internal magnetic field measurement [21].

In the future, the 2-D code can and will be used to design new experiments such as imaging reflectometry experiments [22], poloidal scattering experiments [23] and to study the sensitivity to poloidal correlation lengths. Moreover, an investigation is underway to study the difference between homodyne and heterodyne detection systems from statistical ensembles that are calculated with the $2-\mathrm{D}$ code.

This work was supported by DOE Contract No. DE-AC02-76CH0373. 


\section{References}

[1] P. Cripwell, A.E. Costley, and A.E. Hubbard, 1989 Proceedings of the 16th European Conference on Controlled Fusion and Plasma Physics 13B, part I, 175

[2] R. Nazikian, K. Shinohara K, R. Yoshino, T. Fujita, G.J. Kramer, and the JT-60 team, 1998 IAEA-CN-69/PDP/03 paper presented at 17th IAEA Conf. on Fusion Energy, Yokohama.

[3] M. Gilmore, W.A. Peebles, and X.V. Nguyen, Plasma Phys. Control. Fusion 42 (2000) L1.

[4] M. Gilmore, W.A. Peebles, and X.V. Nguyen, Rev. Sci. Instrum. 72 (2001) 293.

[5] Nazikian R, Kramer G J, Valeo E, Phys. Plasmas 8 (2001) 1840

[6] E. Valeo, G.J. Kramer, and R. Nazikian, Plasma Phys. Control. Fusion 44 (2002) L1.

[7] N. Bretz, Phys. Fluids B 4 (1992) 2414

[8] G.J. Kramer, R. Nazikian, and E. Valeo, Plasma Phys. Control. Fusion 44 (2002) L11.

[9] M. Gilmore, W.A. Peebles, and X.V. Nguyen, Plasma Phys. Control. Fusion 42 (2000) 655.

[10] Joseph W. Goodman, Statistical Optics, John Wiley \& Sons, Inc. (1985).

[11] R. Nazikian, Journal of modern optics 44 (1997) 1037,

[12] R. Nazikian et al., to be published.

[13] W. Gekelman, H. Pfister, Z. Lucky, J. Bamber, D. Leneman, and J. Maggs, Rev. Sci. Instrum. 62 (1991) 2875

[14] F.M. Levinton, Rev. Sci. Instrum. 63) (1992) 5157.

[15] F.M. Levinton, S.H. Batha, Y. Yamada, and C. Zarnstorff, Phys. Fluids B5 (1993) 2554.

[16] R.V. Budny, to be published.

[17] N.L. Bretz, R. Nazikian, and K.L. Wong, Proceedings of the 17th European Physical Society Conference on Controlled Fusion and Plasma Heating, Amsterdam, June 25-29 (1990) 1544. 
[18] R.V. Bravenec, K.W. Gentle, B. Richards, D.W. Ross, D.C. Sing, A.J. Wooton, D.L. Brower, N.C. Juhmann jr., W.A. Peebles, C.X. Yu, T.P. Crowley, J.W. Heard, R.L. Hickok, P.M. Schoch, and X.Z. Yang, Phys. Fluids B 4 (1992) 2127.

[19] R.J. Fonck, N. Bretz, G. Cosby, R. Durst, E. Mazzucato, R. Nazikian, S. Paul, S. Scott, W. Tang, and M. Zarnstorff, Plasma Phys. Control. Fusion 34 (1992) 1993.

[20] R.J. Fonck, G. Cosby, R.D. Durst, S.F. Paul, N. Bretz, S. Scott, E. Synakowski, and G. Taylor, Phys. Rev. Lett. 70 (1993) 3736.

[21] M. Gilmore, private communication.

[22] T. Munsat et al., this conference.

[23] T.L. Rhodes, private communication. 


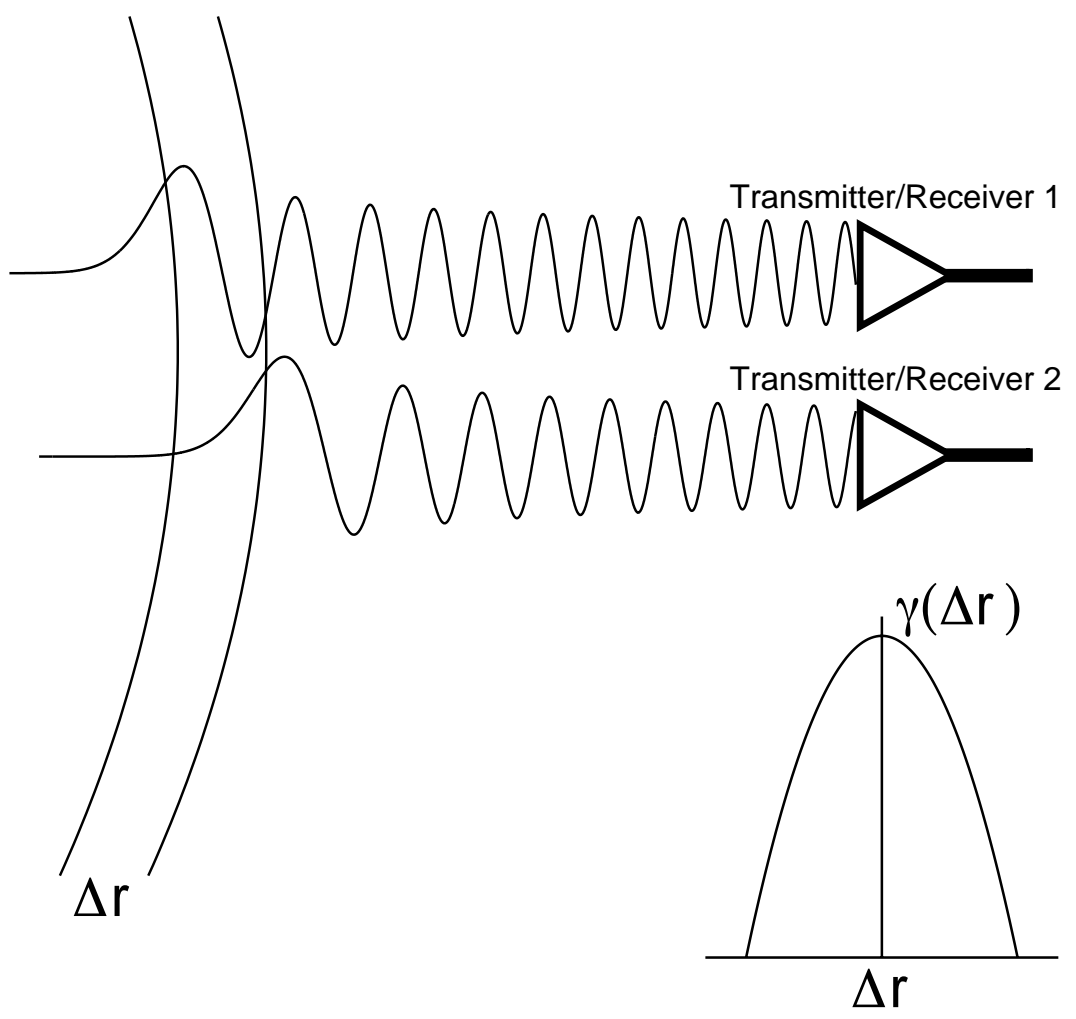

Figure 1: Schematic of correlation reflectometry. Microwaves reflect from different layers, separated by $\Delta r$, in the plasma. When one of the reflectometer frequencies is varied, the cross correlation, $\gamma$, between the signals can be obtained as a function of $\Delta r$ whereby the radial separation is determined from diagnostics that measure the density (X-and O-mode) and magnetic field (X-mode) profiles. 


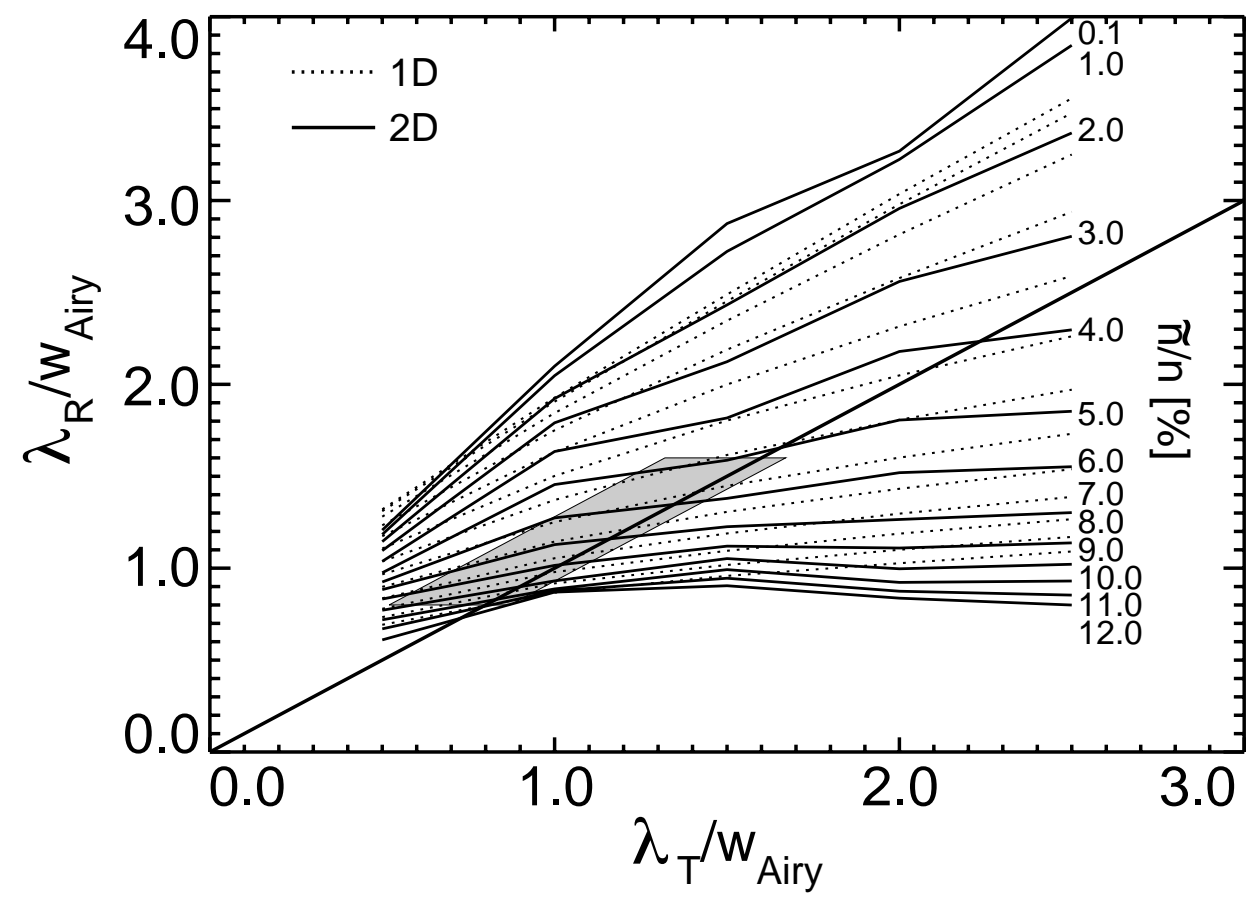

Figure 2: The reflectometer correlation length, $\lambda_{R}$, as a function of the turbulence correlation length, $\lambda_{T}$, for various values of the fluctuation level, $\tilde{n} / n$. The measurements from the LAPD experiments [3] fall within the gray box. 


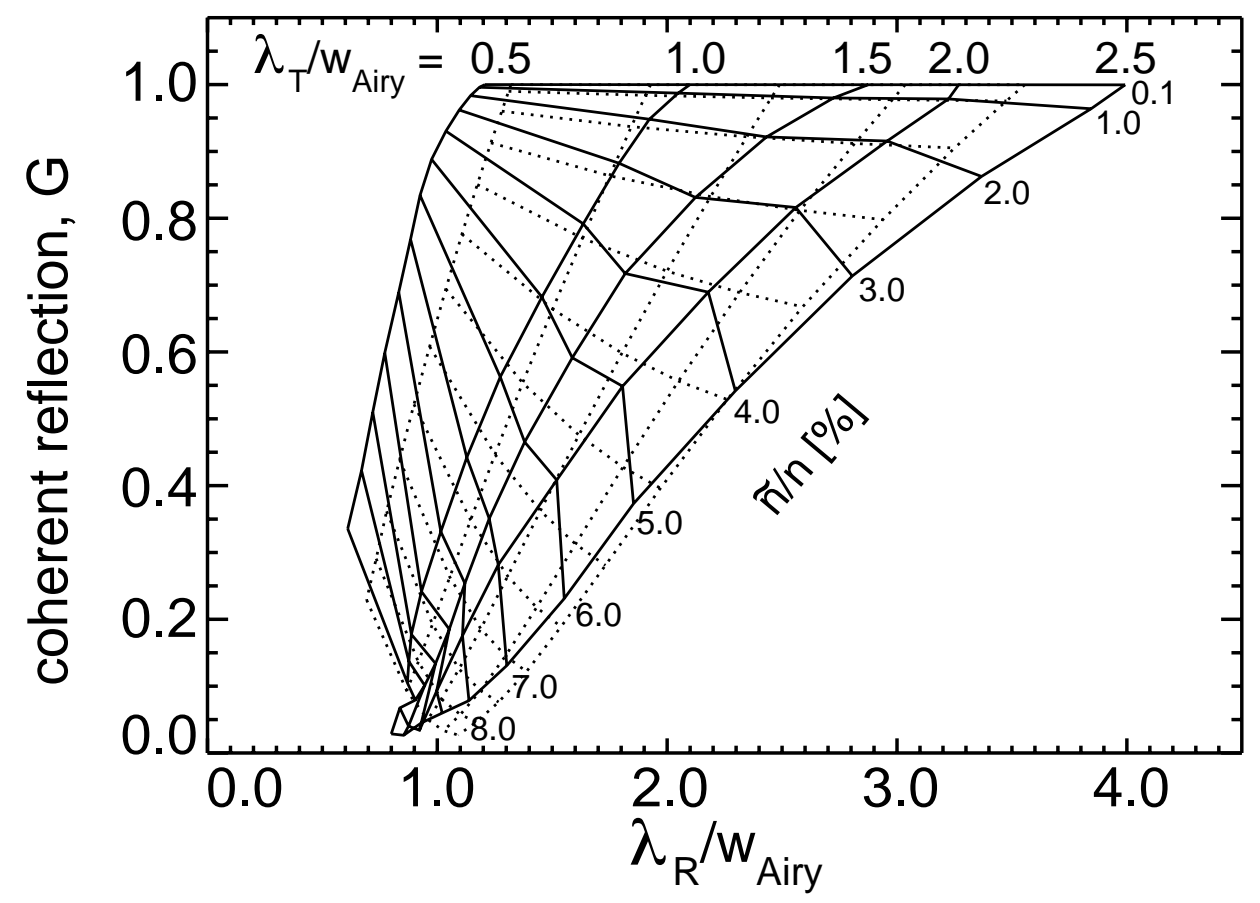

Figure 3: the mapping of the turbulence parameters, turbulence correlation length, $\lambda_{T}$, and fluctuation level, $\tilde{n} / n$, to the experimentally determined quantities, reflectometer correlation length, $\lambda_{R}$, and coherent reflected power $G$. $\lambda_{T}$ and $\lambda_{R}$ are normalized to the Airy width, $W_{\text {airy }}$, of the last fringe at the cut off. The full lines are 2-D and the dotted lines are 1-D simulations. 


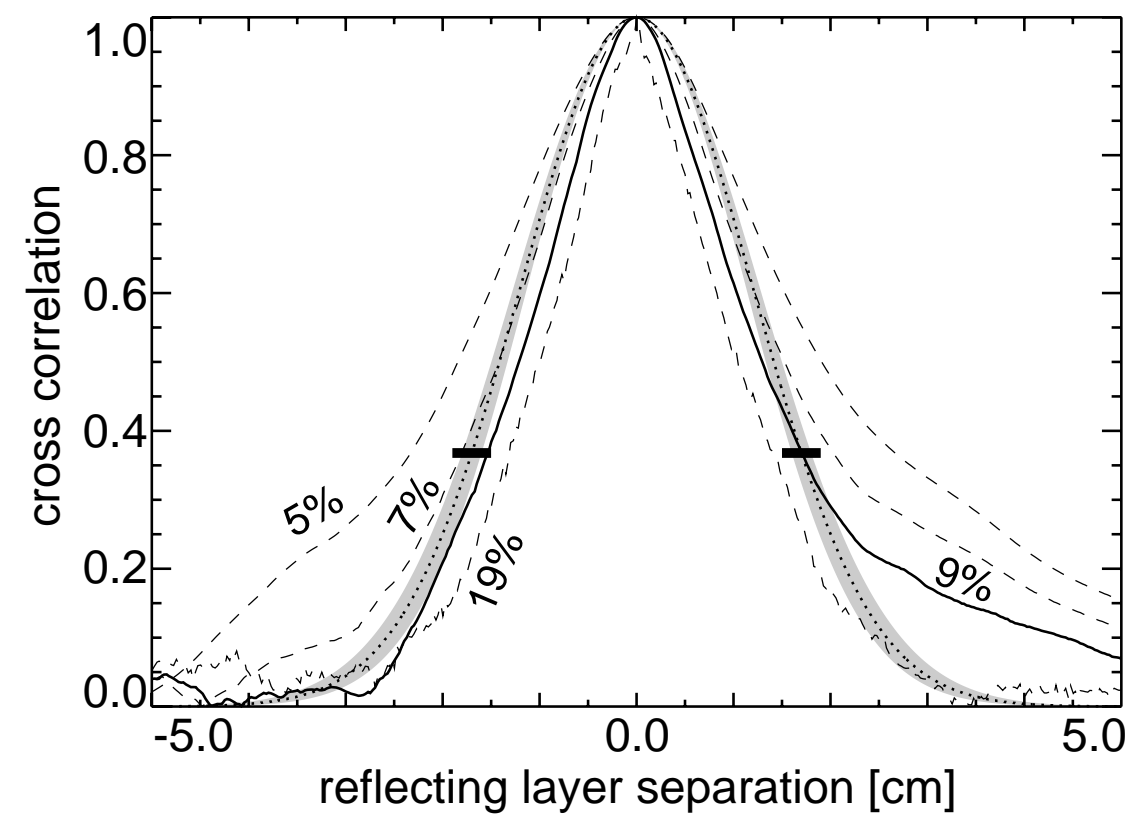

Figure 4: Calculated cross correlation for a LAPD experiment where both the turbulence correlation length and fluctuation level were measured with probes (dotted line, uncertainty: shaded area) and the reflectometer response a the measured fluctuation level of $9 \%$ (solid line). The measured reflectometer 1/e width and its uncertainty is indicated with the error bars at 1 /e and agrees very well with the simulation. For comparison the three dashed curves are the simulated responses at $\tilde{n} / n=5,7$, and $19 \%$. 


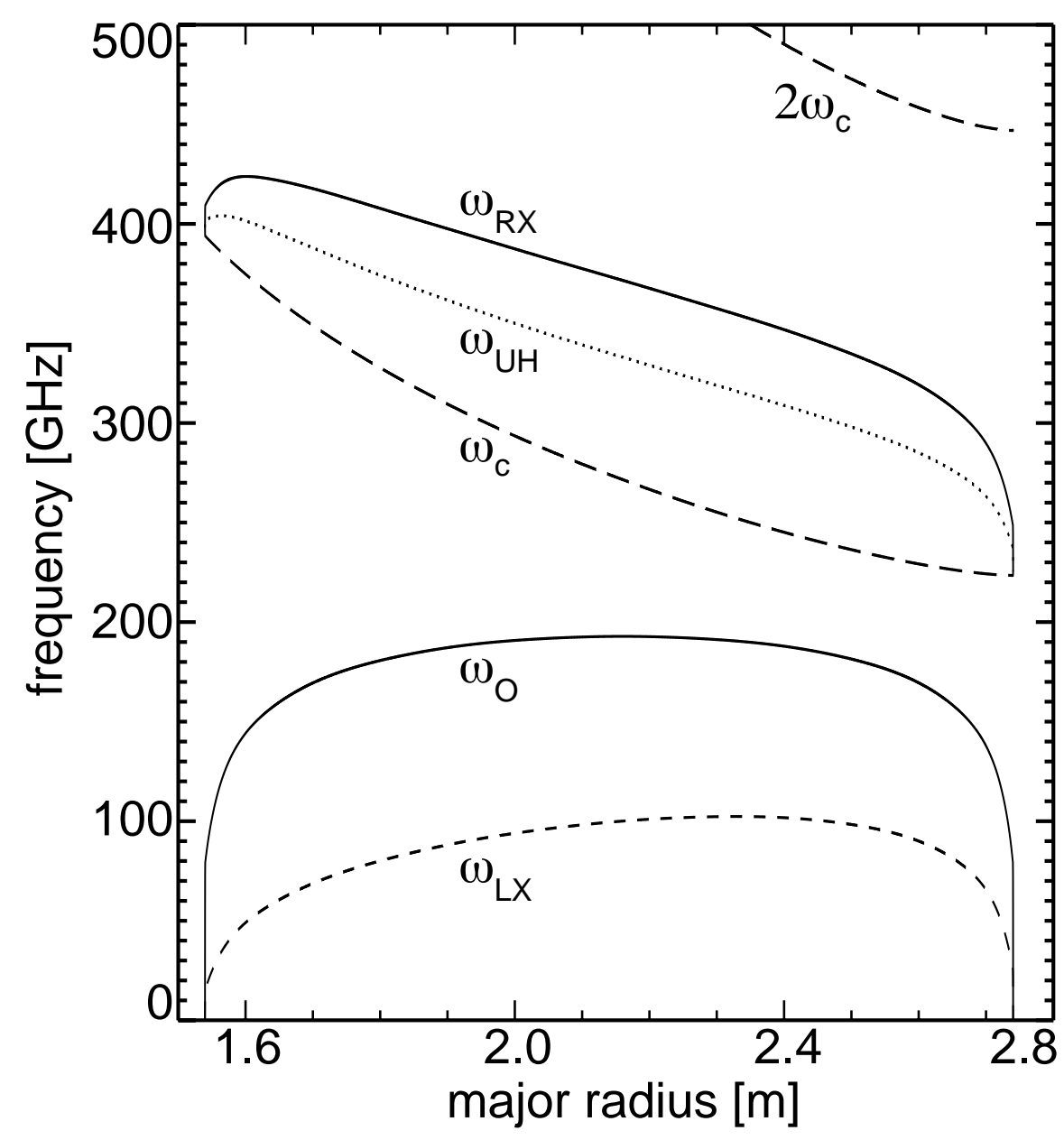

Figure 5: Frequency profiles for various resonant and cut off layers from the FIRE equilibrium used for the O-X correlation study. From bottom to top: $\omega_{\mathrm{LX}}$ the left-hand side X-mode cut off, $\omega_{\mathrm{O}}$ the O-mode cut off, $\omega_{\mathrm{c}}$ the cyclotron resonance, $\omega_{\mathrm{UH}}$ the upper hybrid resonance, $\omega_{\mathrm{LX}}$ the right-hand side X-mode cut off, and $2 \omega_{\mathrm{c}}$ the second harmonic cyclotron resonance. 


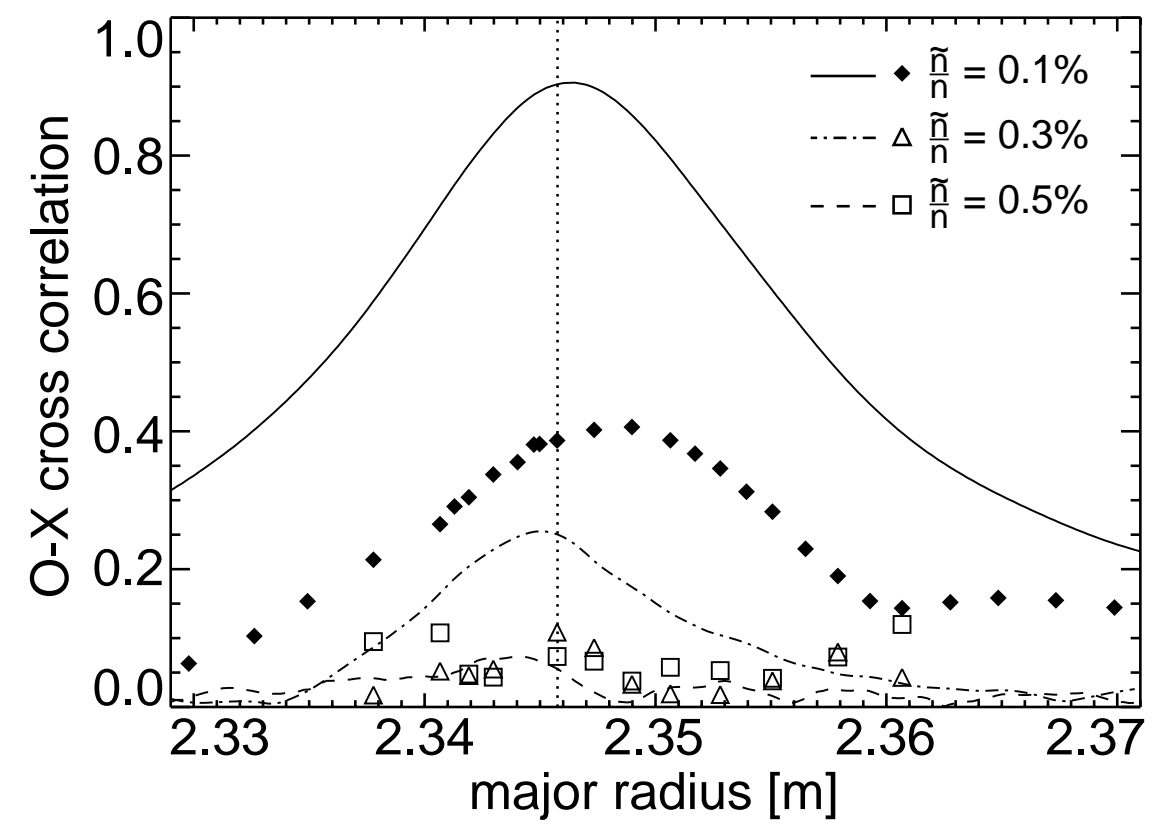

Figure 6: 1-D and 2-D O-X correlation simulations for the standard FIRE equilibrium at different fluctuation levels. The solid, dash dotted, and dashed lines are $1-\mathrm{D}$ simulations for $\tilde{n} / n=0.1,0.3$, and $0.5 \%$, respectively, whereas the diamonds, triangles, and squares are the 2-D simulations for the afore mentioned fluctuation levels. The vertical dotted line is the 1-D O-mode reflection location at $190 \mathrm{GHz}$. 


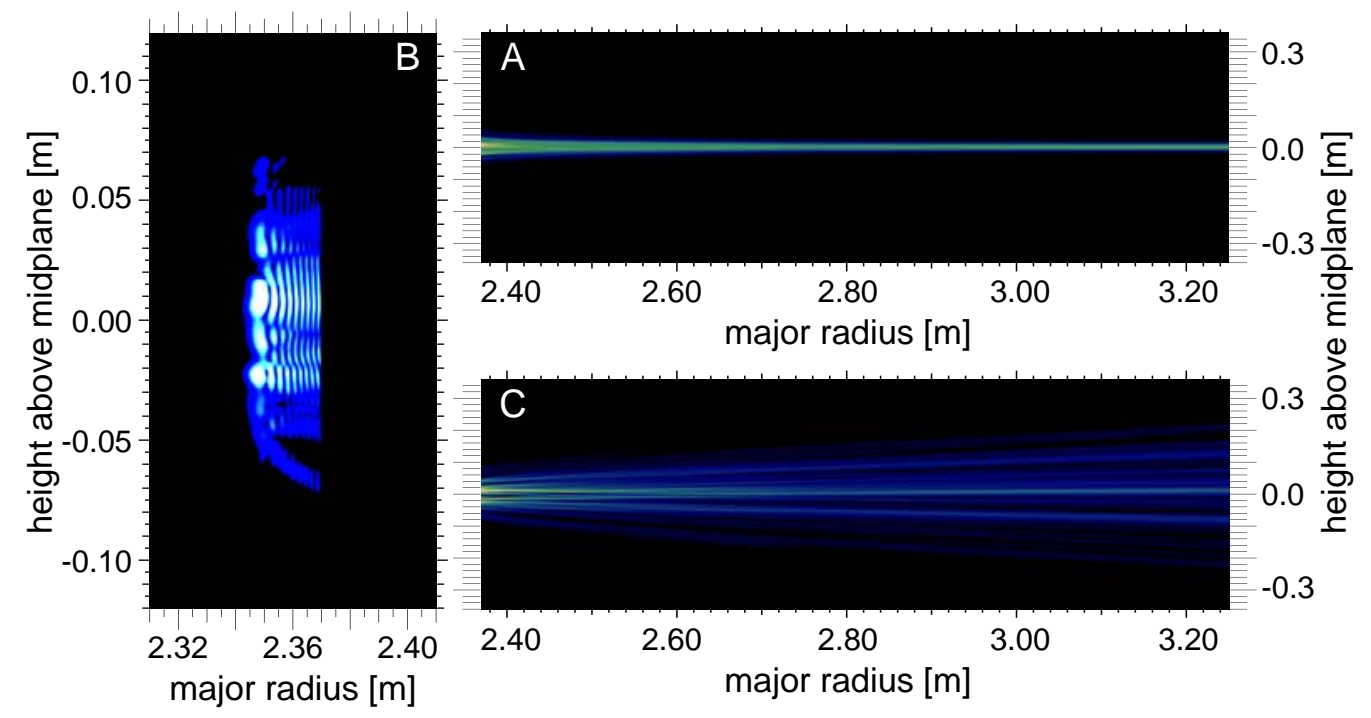

Figure 7: The intensity of the electric field for $352.6 \mathrm{GHz}$ X-mode waves propagating in the plasma. (A) the paraxial solution of the incoming waves, (B) the full wave solution near the cut off, and (C) the paraxial solution of the outgoing waves. Note the difference in vertical scale between this figure and figure 8 . 


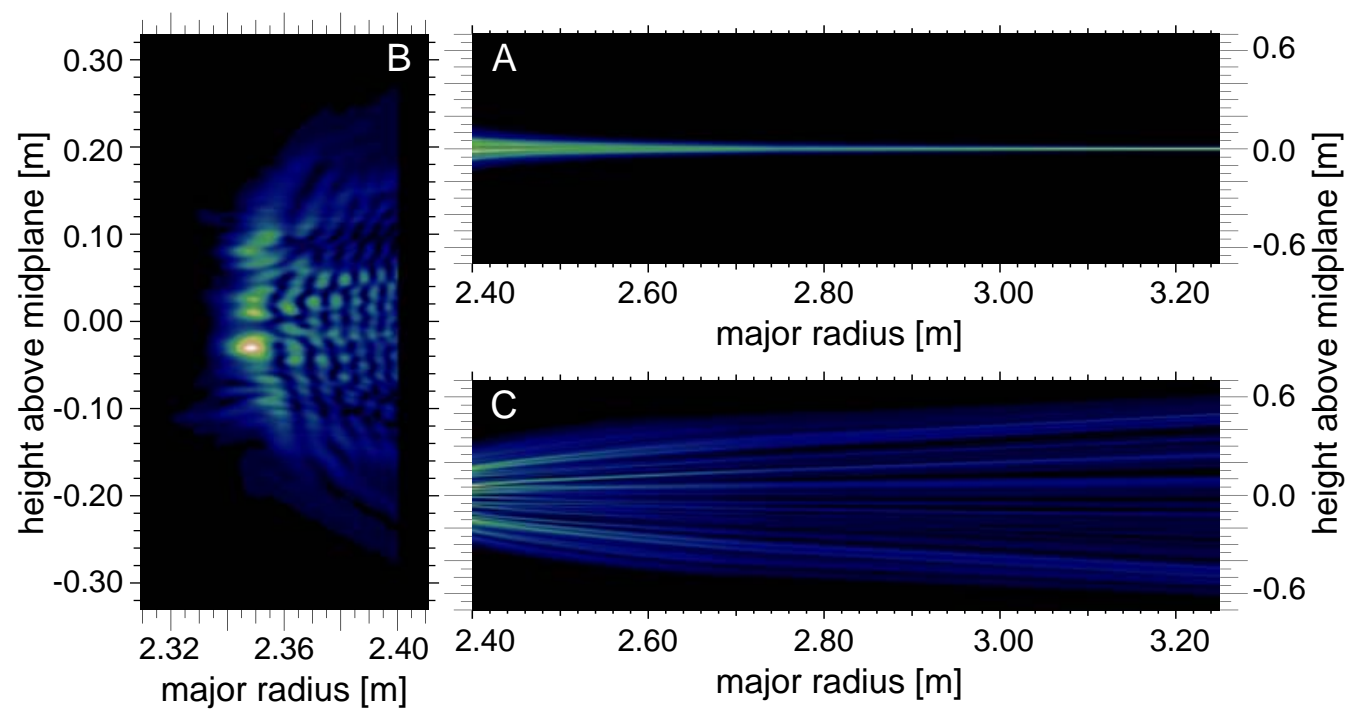

Figure 8: The intensity of the electric field for $190.0 \mathrm{GHz}$ O-mode waves propagating in the plasma. (A) the paraxial solution of the incoming waves, (B) the full wave solution near the cut off, and (C) the paraxial solution of the outgoing waves. Note the difference in vertical scale between this figure and figure 7 . 


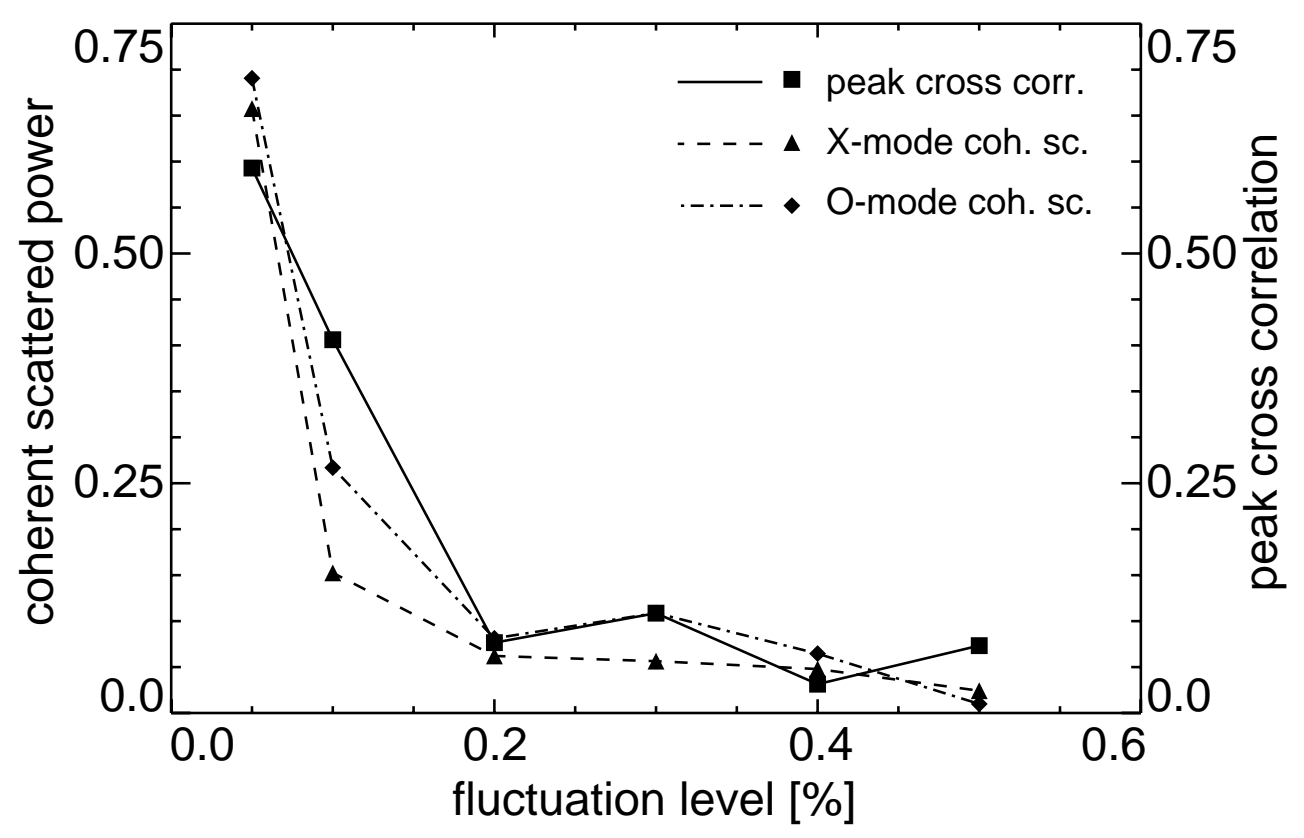

Figure 9: Peak O-X cross correlation (solid line) and coherent scattered power for $\mathrm{X}$-mode (dashed line) and 0-mode (dashed dotted line) as a function of density fluctuation level for the FIRE equilibrium at $r / a=0.34$. 


\section{External Distribution}

Plasma Research Laboratory, Australian National University, Australia

Professor I.R. J ones, Flinders University, Australia

Professor J oão Canalle, Instituto de Fisica DEQ/IF - UERJ , Brazil

Mr. Gerson O. Ludwig, Instituto Nacional de Pesquisas, Brazil

Dr. P.H. Sakanaka, Instituto Fisica, Brazil

The Librarian, Culham Laboratory, England

Library, R61, Rutherford Appleton Laboratory, England

Mrs. S.A. Hutchinson, JET Library, England

Professor M.N. Bussac, Ecole Polytechnique, France

Librarian, Max-Planck-Institut für Plasmaphysik, Germany

J olan Moldvai, Reports Library, MTA KFKI-ATKI, Hungary

Dr. P. Kaw, Institute for Plasma Research, India

Ms. P.J . Pathak, Librarian, Insitute for Plasma Research, India

Ms. Clelia De Palo, Associazione EURATOM-ENEA, I taly

Dr. G. Grosso, Instituto di Fisica del Plasma, Italy

Librarian, Naka Fusion Research Establishment, J AERI, J apan

Library, Plasma Physics Laboratory, Kyoto University, J apan

Research Information Center, National Institute for Fusion Science, J apan

Dr. O. Mitarai, Kyushu Tokai University, J apan

Library, Academia Sinica, Institute of Plasma Physics, People's Republic of China

Shih-Tung Tsai, Institute of Physics, Chinese Academy of Sciences, People's Republic of China

Dr. S. Mirnov, TRINITI, Troitsk, Russian Federation, Russia

Dr. V.S. Strelkov, Kurchatov Institute, Russian Federation, Russia

Professor Peter Lukac, Katedra Fyziky Plazmy MFF UK, Mlynska dolina F-2, Komenskeho Univerzita, SK-842 15 Bratislava, Slovakia

Dr. G.S. Lee, Korea Basic Science Institute, South Korea

Mr. Dennis Bruggink, Fusion Library, University of Wisconsin, USA

Institute for Plasma Research, University of Maryland, USA

Librarian, Fusion Energy Division, Oak Ridge National Laboratory, USA

Librarian, Institute of Fusion Studies, University of Texas, USA

Librarian, Magnetic Fusion Program, Lawrence Livermore National Laboratory, USA

Library, General Atomics, USA

Plasma Physics Group, Fusion Energy Research Program, University of California at San Diego, USA

Plasma Physics Library, Columbia University, USA

Alkesh Punjabi, Center for Fusion Research and Training, Hampton University, USA

Dr. W.M. Stacey, Fusion Research Center, Georgia Institute of Technology, USA

Dr. J ohn Willis, U.S. Department of Energy, Office of Fusion Energy Sciences, USA

Mr. Paul H. Wright, Indianapolis, Indiana, USA 
The Princeton Plasma Physics Laboratory is operated by Princeton University under contract with the U.S. Department of Energy.

\author{
Information Services \\ Princeton Plasma Physics Laboratory \\ P.O. Box 451 \\ Princeton, NJ 08543
}

Phone: 609-243-2750

Fax: 609-243-2751

e-mail: pppl_info@pppl.gov

Internet Address: http://www.pppl.gov 